\title{
System-Level Estimation of Threshold Voltage Degradation due to NBTI with I/O Measurements
}

\author{
Soonyoung Cha, Chang-Chih Chen, and Linda S. Milor \\ Georgia Institute of Technology, 30332, Atlanta, GA, USA, E-mail: scha31@gatech.edu
}

\begin{abstract}
With the scaling of CMOS technology, Negative Bias Temperature Instability (NBTI) and Process Variations (PV) are serious issues for transistors. Normally, degradation due to NBTI is modeled based on test structure data or ring oscillators embedded within product die. In this paper, we present a method to determine the initial average channel length $(L)$ and threshold voltage $\left(V_{t h}^{0}\right)$ for individual chips, together with NBTI model parameters through $\mathrm{I} / \mathrm{O}$ measurements. We determine a relationship between $\Delta V_{t h}, V_{t h}^{0}, L$ and ground signal variation and fit models to the simulation results. The voltage of the ground signal is used for the calculation of the delay and amplitude shifts which are used to extract PV and NBTI parameters. Then, we calculate the lifetime for each chip individually using calibrated NBTI models, accounting for process variations. The methodology enables the extraction of NBTI and PV model parameters for individual chips, not just for the manufacturing process, and hence it becomes possible to differentiate chips that have different parameters initially and are more or less vulnerable to NBTI.
\end{abstract}

\section{INTRODUCTION}

NBTI is an important wearout mechanism, which affects pMOS devices by shifting the threshold voltage $\left(V_{t h}\right)$. This increase in $V_{t h}$ degrades the operating speed of circuits, and, in extreme cases, causes the circuit to behave incorrectly because of timing constraints [1]. Also, difficulties in controlling lithography and doping cause significant PV. Because PV also cause variation in the transistor characteristics (channel length, threshold voltage, oxide thickness), which adds to that caused by NBTI, chip reliability should take into account not only the NBTI-induced $V_{t h}$ shift, but also the initial $V_{t h}$ and channel length in each individual chip [2].

During the last decade, the reaction-diffusion (R-D) model has been used to explain the NBTI effect in a pMOS device, which is the breaking and rebonding of hydrogen-silicon bonds at the silicon-gate dielectric interface [3]. However, although the R-D model explains the threshold voltage shifts produced by $\mathrm{DC}$ bias and temperature stress, it could not explain the speed of recovery and the impact of the dynamic gate bias [4][8]. Therefore, recently, the charge trapping and detrapping (TD) model is being considered as a more likely explanation of the NBTI mechanism [4],[5].

Starting with a physical model of NBTI, which characterizes the threshold voltage shift as a function of time, the device-level models can be used to derive gate-level simulation models and system lifetime [9]-[12]. System-level prediction of lifetime enables the optimization of timing guardbands or circuit adaptation based on a prediction of the increase in delay as a function of time, temperature, and usage. This can be done with look-up tables of frequency and voltage pairs as a function of time under operation [13]. This approach assumes that the NBTI model is known, and hence errors result if failure rate parameters or operating history are not estimated correctly. Also, because the lifetime of a circuit is jointly affected by NBTI and PV, circuit timing has to consider both effects.

Alternatively, ring oscillators [14] or replica critical paths [15],[16] are used to analyze degradation. However, the ring oscillators or the replica critical paths do not share the same ambient environment because of the difference in their on-die location and the critical paths within a datapath can change as a result of degradation [17]. Hence, this approach may not produce an accurate prediction of the aging rate of the true critical paths. An alternative, involving direct monitoring of critical paths [18],[19], entails some area overhead and the correct selection of the vulnerable critical paths. Another approach involving direct monitoring of aging, involves the use of specially designed latches that detect delay errors, at the expense of significant power overhead per latch [20],[21].

In this paper, we build a method to find initial average PV parameters using the ground voltage signal. We analyze the relationship between PV parameters and an initial signature signal at time zero to estimate the avearge values for PV parameters. Also, as mentioned in [22], the threshold voltage shift due to NBTI translates into shifts in current, which should be visible by monitoring the signature on the ground terminal during operation. We find a relationship between the $V_{t h}$ shift and the shift in the signature to extract NBTI parameters. In an actual circuit, data are measured at the I/Os using the ground signature. PV parameters are calculated using the initial ground signal, and the NBTI parameters are derived from the threshold voltage shift, extracted from the shift in the ground signal with time. The results are combined to provide a lifetime estimate for each chip. Fig. 1 shows the full process of how we can get the PV and NBTI model for a circuit using measurement data from the ground signature and how we can use two models, one for the NBTI failure rate parameters and one for PV, to estimate the lifetime for each chip, accounting for PV.

We summarize, in Section II, the T-D model of the average and standard deviation of the threshold voltage shift. In Section III, the system level chip simulation results are generated by FastSpice. For the PV effect on a chip, we consider the global variation of $V_{t h 0}$ and the length of MOS devices for the whole chip. Then, we extract the signature signal, which is affected by variation. Using the affected signature signal and comparing it with the nominal signature signal, we can extract the delay and amplitude variation. In order to see the degradation effect, we applied the $\mu\left(\Delta V_{t h}\right)$ and $\sigma\left(\Delta V_{t h}\right)$, extracted in Section II to each stressed pMOS to determine the degradation of the signature signal. The signature signal is analyzed to extract the amplitude and delay shift. We check the relationship between the threshold voltage shifts and amplitude and delay shifts for different circuits and input patterns. Then, we determine the accuracy of our model 


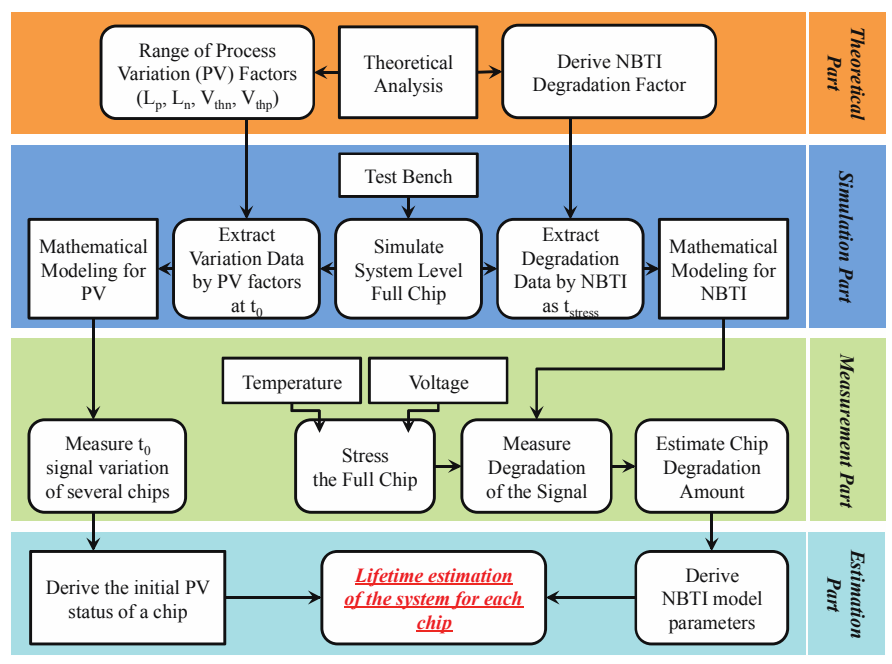

Fig. 1. The schematic of the proposed flow to find the PV status, NBTI degradation model, and the lifetime of each chip.

for PV extraction and NBTI degradation. Section IV presents the experimental results for extracting PV and the $V_{t h}$ shift for the same chips as a function of stress time. Then, we estimate the lifetime of each chip, based on the extracted model parameters and conclude with a summary in Section V.

\section{Charge Trapping ANd Detrapping NBTI MOdel}

Random Telegraph Noise (RTN), that is, charge trapping and detrapping of oxide defects, has been observed in submicron FETs. Defects, which are located in the oxide layer in pMOSs, capture and emit charges [23]. If a defect captures a charge carrier in the oxide $\left(\mathrm{SiO}_{2}\right)$, unexpected current noise occurs, and the charged defects affect the mobility and scattering in the device [23],[24].

For a device under constant bias, because charge trapping depends on the Fermi level only [24], the current remains constant on average and does not change with time. In digital IC applications, the gate voltage (bias) and the Fermi level change abruptly because of the digital input signals. This increases the probability of trapping, and the number of captured charges increases over time. Therefore, the gradually increasing number of occupied traps changes the channel conductivity, which is a source of bias temperature instability.

In the T-D model, the number of defects, which trap charge has a functional relationship with bias voltage, temperature $(T)$, and time $(t)$. These defects lead to an average and standard deviation of threshold voltage degradation. First, the number of defects undergoing capture and reemission is modeled with the Poisson distribution with time constants corresponding to emission and capture [25]. The time constants are random variables that depend on temperature, bias point and the trapping speed of defects [26]. The time constants are uniformly distributed on the log scale and the distribution is linked to the Fermi energy level and temperature [27]. The number of defects, $n(t)$, for a specific device is distributed according to a Poisson distribution [28]. The number of defects is integrated over the time constants to give [29]:

$$
\begin{gathered}
n(t) \sim \varphi\left(T, E_{F}\right)(A+B \log (t)) \\
\sigma(n(t)) \sim \varphi\left(T, E_{F}\right) \sqrt{A+B \log (t)}
\end{gathered}
$$

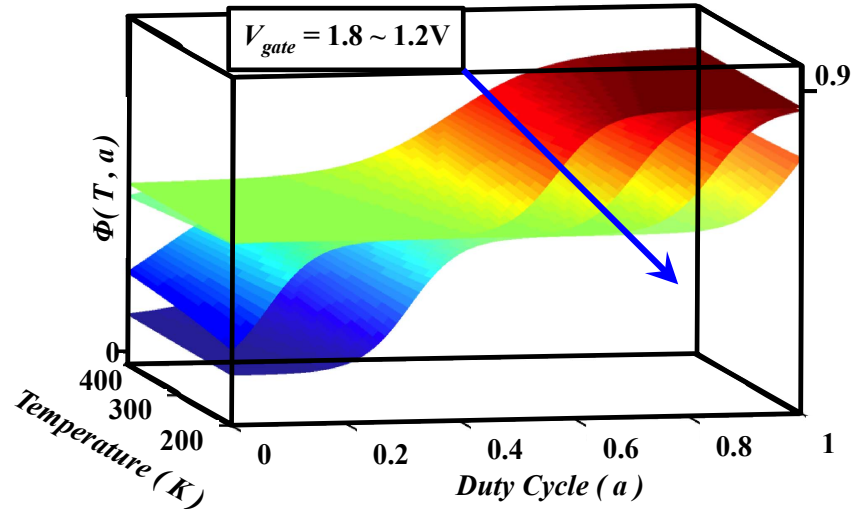

Fig. 2. The dependence of the threshold voltage shift on the duty cycle and temperature. The threshold voltage is a linear function of $\phi(T, \alpha)$, as noted in equations (1) and (3), where $\Delta V_{t h}=\delta \phi\left(T, E_{F, e f f}\right)(A+B \log (t))$ and where $E_{F, e f f}$ is a function of $\alpha$, according to equation (4).

where $\mathrm{A}$ and $\mathrm{B}$ are constants, $t$ is a stress duration and should be larger than 0 , and $\varphi\left(T, E_{F}\right)$ is a function that depends on the trap energy density distribution in the band-gap. Note that equation (1) applies to both the stress and recovery phases, and the Fermi level models the impact of the applied voltage.

The fluctuation in average threshold voltage is determined by multiplying equation (1) by $\delta$, the shift in threshold voltage due to a single trap [30], to get

$$
\Delta V_{t h}(t)=\delta n(t)
$$

Therefore, using equation (3), we obtain the theoretical average and standard deviation of the shift in threshold voltage for each pMOS device in our system. Note that the relative variation in the number of defects, $\sigma(n(t)) / n(t)$ decreases as a function of time, as does the relative variation in the threshold voltage. This is consistent with experimental observations of delay variations in circuits [31].

Digital circuit operation has stress and recovery periods. The fraction of time under stress is called the duty cycle, $\alpha$. Instead of modeling the shift in threshold voltage for each stress and recovery period separately, it is best to determine an effective Fermi level, as a function of duty cycle [32]:

$$
E_{F, e f f}(\alpha)=\alpha E_{F, \text { on }}+(1-\alpha) E_{F, \text { off }}
$$

The effective Fermi level is used to determine the evolution of threshold voltage degradation. Therefore, $\varphi\left(T, E_{F, \text { eff }}\right)$ can more reasonably adjust the probability of charge capture and emission. For example, it can be seen that as $\alpha$ approaches one, when there is no time for recovery, degradation is much more significant. This is illustrated in Fig. 2, using the Ushaped trap density distribution function for $\varphi(T, \alpha)$ [23],[29].

Fig. 3 illustrates the shift in pMOS threshold voltage for a switching inverter, showing stress and recovery cycles. It also illustrates a ground signature signal, which shows the shift in delay and amplitude as a function of time under stress.

\section{Signatrue Signal ANALYSIS AND Modeling for NBTI AND PROCESS VARIATION}

Several case studies involving different chips and test benches have been considered: a Microprocessor, a Floating Point Unit (FPU), and a Finite Impulse Response (FIR) filter. 


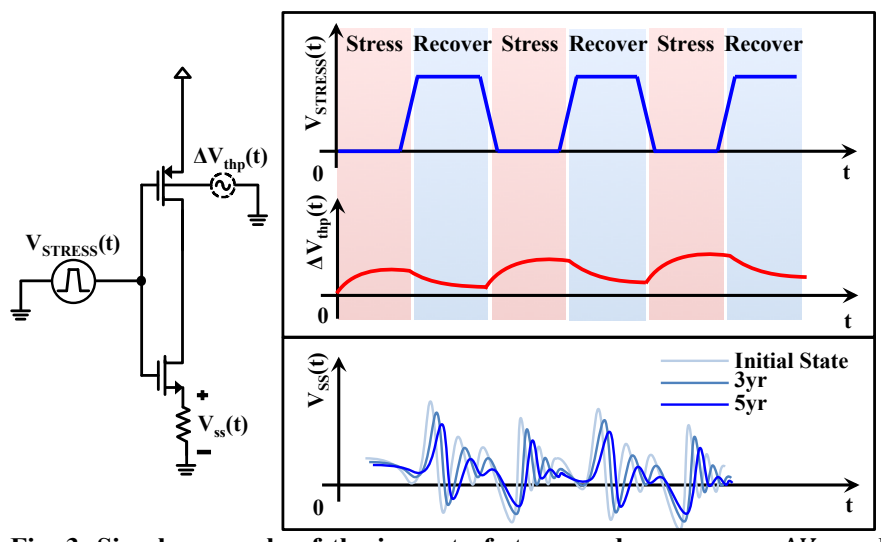

Fig. 3. Simple example of the impact of stress and recovery on $\Delta V_{t h}$ and the corresponding ground signal, Vss(t), at different time points.

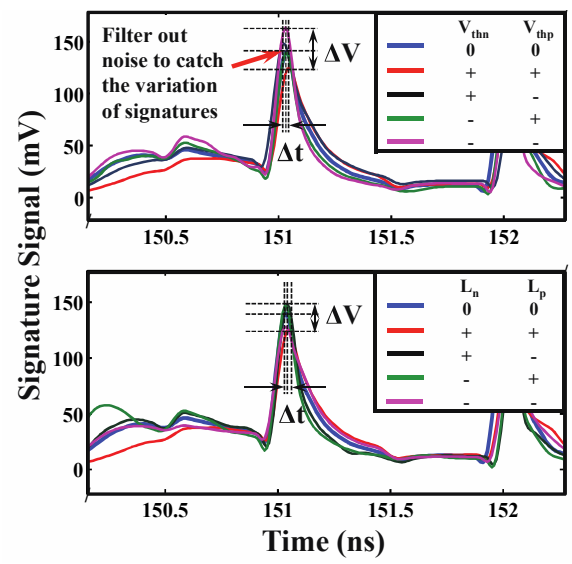

Fig. 4. Signature signal variation caused by process variation.

The Microprocessor is a RISC processor with an external memory. It has around 1.4 million pMOSs. The FPU and FIR filter have $20 \mathrm{k}$ and $8 \mathrm{k}$ pMOSs, respectively. We simulate each of these systems with SPICE to obtain the ground voltage signature. The test benches determine the stress of each transistor. Hence, variation among the test benches provides an indication of variation due to use conditions. For example, for the microprocessor, the shift is largest for the ADD testbench and the least for the CACHE testbench.

For PV, we model only the average initial threshold voltage $\left(V_{t h}^{0}\right)$ and channel length $(L)$, for pMOS and nMOS devices, since these factors are not controlled well by the process and they are important for timing. In addition to the random variation in the initial threshold voltage values, NBTI causes the mean and standard deviation of the threshold voltages of pMOSs to increase. The amount of the increase was modeled in the previous section, where we found the relationship between time and $\mu\left(\Delta V_{t h 0}\right)$ and $\sigma\left(\Delta V_{t h 0}\right)$. Based on these values and an initial Gaussian distribution, we obtain the threshold voltage distribution for each pMOS to generate the virtual NBTI effect.

\section{A. Data Extraction and Modeling for PV}

In this work, we consider the ground voltage signature. Fig. 3 shows the ground voltage signature as a function of the threshold voltage shift. Fig. 4 shows an example signature, which is affected by PV. The delay and amplitude variation are compared to the nominal signature signal, where $\Delta L=0$ and

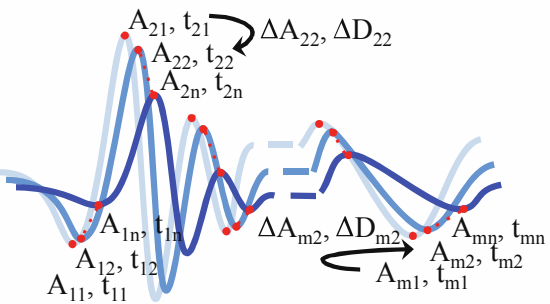

Fig. 5. Finding the amplitude and delay points in the ground signature.

$V_{t h}^{0}=0$. In the figure, in order to generate this result, process parameters were varied in accordance with a full factorial experimental design [33], with high and low values represented by + and - in the graph. It can be seen that PV affects the amplitude and delay of the peaks of the ground signal.

The process of extracting PV involves three steps. First, we use a full factorial design to generate a set of 16 experiments at the 16 corners of the process domain. In other words, a maximum and minimum value is set for the nMOS and pMOS threshold voltage domain and the nMOS and pMOS channel length domain. Simulations of the ground signatures are performed for each combination of the corners for $V_{t h p 0}, V_{t h n 0}$, $L_{p 0}$, and $L_{n 0}$. This consists of a 16 simulations.

Second, the signature on the ground terminal is computed for an input pattern. Each maximum and minimum point in the signature is determined and associated with an amplitude and time point, shown in Fig. 5. Hence, several amplitude and time point pairs are computed for each signature signal. Third, we calculate the difference between the time point and amplitude pairs for the nominal signal and the corner signal to form a matrix. The matrix has 16 rows corresponding to 16 corner simulations and one column for each peak point in the signature.

Each signature has many peaks. Hence, the shifts in the peaks produce a large data set of amplitude and delay shifts $\left(A_{P V}\right.$ and $\left.D_{P V}\right)$, which is reduced to a few principal components [34]. The principal components are linear combinations of the shifts in delay and amplitude, i.e. $P C_{i}=\sum_{j} a_{i j} \Delta A_{j}+b_{i j} \Delta D_{j}$. They provide dimensional reduction and are computed using the covariance matrix among the shifts in amplitude and delay at the set of peaks. The values of the principal components are computed for each of the 16 corner experiments. From this set of experiments, each principal component is modeled as a function of PV, i.e. the shift from an assumed nominal value for channel length and threshold voltage, $\Delta L$ and $\Delta V_{t h}^{0}$ for nMOS and pMOS devices, i.e. $P C_{i}=c_{i 1} \Delta L_{n}+c_{i 2} \Delta L_{p}+$ $c_{i 3} \Delta V_{t h n}^{0}+c_{i 4} \Delta V_{t h p}^{0}$. If there are at least four principal components, then it becomes possible to solve for the average shift in $\Delta L_{n}, \Delta L_{p}, \Delta V_{t h n}^{0}$, and $\Delta V_{t h p}^{0}$. For $m$ principal components, the matrix $C$ is $m \times 4$, where each row corresponds to a principal component. Then, for an unstressed circuit, the difference between a simulated nominal signature and an experimental signature is computed. The shifts in amplitude and delay are computed, $\Delta A_{j}$ and $\Delta D_{j}$. These values are used to computed the corresponding principal components, $P C_{i}, i=1, \ldots, n$, to form a right hand side vector, $P C$. If $C^{T}$ is the transpose of $C$, then we solve for a vector of process variations, $\Delta p$ as the solution of $\Delta p=\left(C^{T} C\right)^{-1} C^{T} P C$. Effectively, the process parameters are estimated as a linear combination of shifts in amplitude and delay, i.e., 
TABLE I.

THE ERror $(\delta)$ OF EACH EXPECTED INITIAL SOURCE IN EQUATION (5)

\begin{tabular}{ccccccc}
\hline \hline \multirow{2}{*}{$\begin{array}{c}\text { Average error rate, } \boldsymbol{\delta}, \\
\text { of each source }(\%)\end{array}$} & \multicolumn{6}{c}{ Applied standard deviation to $\boldsymbol{L}$ and $\boldsymbol{V}_{\boldsymbol{t h}}^{\mathbf{0}}$} \\
\cline { 2 - 7 } $\boldsymbol{\delta}\left(\boldsymbol{\Delta}_{\mathbf{n}}\right)$ & 5.96 & 5.97 & 5.98 & 5.99 & 5.99 & 6.00 \\
\hline $\boldsymbol{\delta}\left(\boldsymbol{\Delta}_{\mathbf{p}}\right)$ & 5.94 & 5.97 & 5.98 & 6.00 & 5.99 & 5.98 \\
\hline $\boldsymbol{\delta}\left(\boldsymbol{\Delta} \boldsymbol{V}_{\text {thn }}^{\mathbf{0}}\right)$ & 3.91 & 3.94 & 3.94 & 3.94 & 3.94 & 3.94 \\
\hline $\boldsymbol{\delta}\left(\boldsymbol{\Delta} \boldsymbol{V}_{\text {thp }}^{\mathbf{0}}\right)$ & 3.22 & 3.22 & 3.22 & 3.22 & 3.23 & 3.22 \\
\hline \hline
\end{tabular}

$\Delta L=\sum_{i=1}^{n}\left(a_{i} \Delta A_{i}+b_{i} \Delta D_{i}\right), \Delta V_{t h}^{0}=\sum_{i=1}^{n}\left(c_{i} \Delta A_{i}^{\prime}+d_{i} \Delta D^{\prime}{ }_{i}\right)$.

\section{B. Validation of the Methodology for Extracting $P V$}

We need to check the validity of our methodology. To mimic a real process, each device is assigned a random initial threshold voltage and channel length. For each sample, an average shift in threshold voltage and channel length for nMOS and pMOS devices is applied. The signature is computed by simulation for each sample and shifts in amplitude and delay are computed for the peak points. From these shifts, the model computed with equation (5) is used to estimate the mean shift in threshold voltage and channel length. The estimation of threshold voltage and channel length is compared with the actual shifted value. Table I shows the average error rate based on several simulation runs.

Within-die random variation can reduce the accuracy in estimating the mean values of threshold voltage and channel length. Hence, we have included the impact of random variation $(0 \sim 10 \%)$ in Table I. All errors are under $6 \%$, indicating that process parameter values can be computed accurately.

\section{Data Extraction and Modeling for NBTI}

Given a ground voltage signature, for the analysis of NBTI, our method extracts the shift in delay and amplitude as a function of time. Instead of collecting a set of signatures from a full factorial experiment, signatures are generated with an initial random set of process parameters, to mimic a true process, at different time points, to mimic the impact of stress. The delay $\left(\Delta D_{N B T I}\right)$ and amplitude $\left(\Delta A_{N B T I}\right)$ sets are computed, at each peak, as illustrated in Fig. 5. The average delay and amplitude as a function of time for NBTI is computed to form the vectors $D_{N B T I}$ and $A_{N B T I}$. Fig. 6(a) shows a signature signal as a function of $V_{t h}$ degradation. Fig. 6(b) shows the delay and amplitude degradation due to NBTI for different circuits and test benches at different time points. We find the average $D_{N B T I}\left(\Delta D_{\text {avg } i}\right)$ and $A_{N B T I}\left(\Delta A_{\text {avg } i}\right)$ shift of peak points in the signature at different stress times, and $\Delta V_{t h}$ is computed by regression [34] from the average shifts in the peak points of the signature, i.e.,

$$
\mu\left(\Delta V_{t h}\right)=\varepsilon+\alpha \Delta A_{\text {avg }}+\beta \Delta D_{\text {avg }}+\gamma \Delta A_{\text {avg }} \Delta D_{\text {avg }} .
$$
where $\alpha, \beta, \gamma$, and $\varepsilon$ are fitting constants. Fig. 6(c) compares the average shift in threshold voltage with the true shift in threshold voltage, showing that our method is accurate. Given an extracted shift in the threshold voltage as a function of time, computed using the model in equation (6), the model parameters in equation (1), $A$ and $B$ are computed.

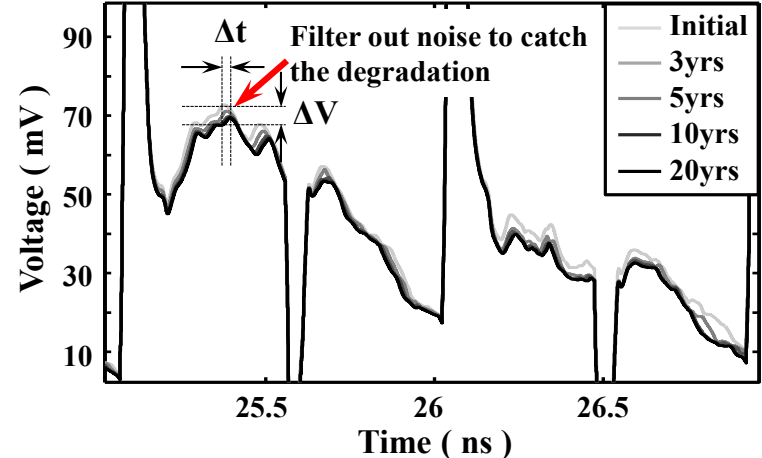

(a)

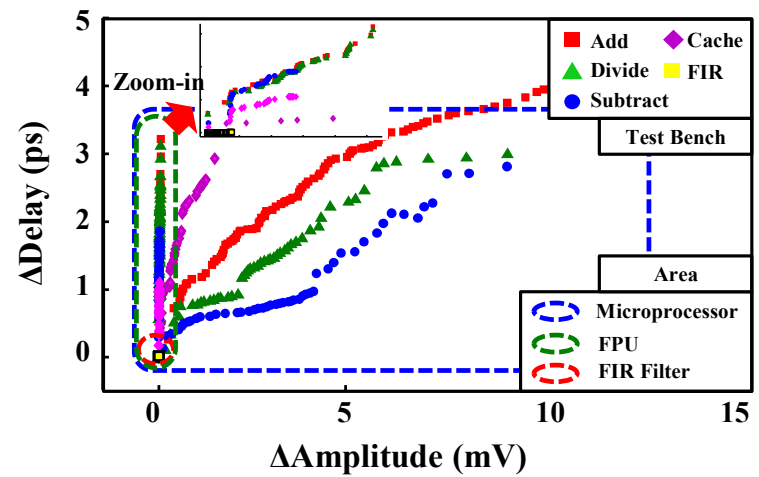

(b)

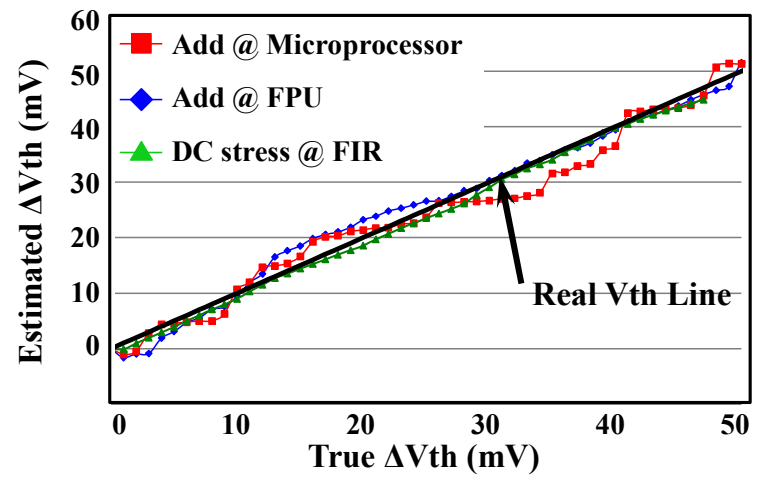

(c)

Fig. 6. Signature signal and regression result for NBTI degradation for different chips. (a) A part of the ground signature signal as a function of the threshold voltage shift. (b) $\Delta$ Delay and $\Delta$ Amplitude data distribution. (c) Model vs. actual threshold voltage shift.

\section{Validation of the Methodology for Extracting NBTI Parameters}

We need to check if our methodology can correctly extract NBTI model parameters in equation (1) using signature data. To do this, we assume process-level values are given for coefficients A and B in (1). Each device is assigned a random initial threshold voltage. NBTI degradation models are applied to each device, and the signature is simulated. From the signature, the shift in amplitude and delay is computed, and the average shift in threshold voltage is estimated. From the shift in threshold voltage as a function of time, we estimate A and B.

Table II shows the average error rate for each coefficient, based on several simulation runs for each of the circuits and test benches using full chip simulations. 
TABLE II.

THE ERRor $(\delta)$ IN COMPUTING THE COEFFICIENTS IN EQUATION (1)

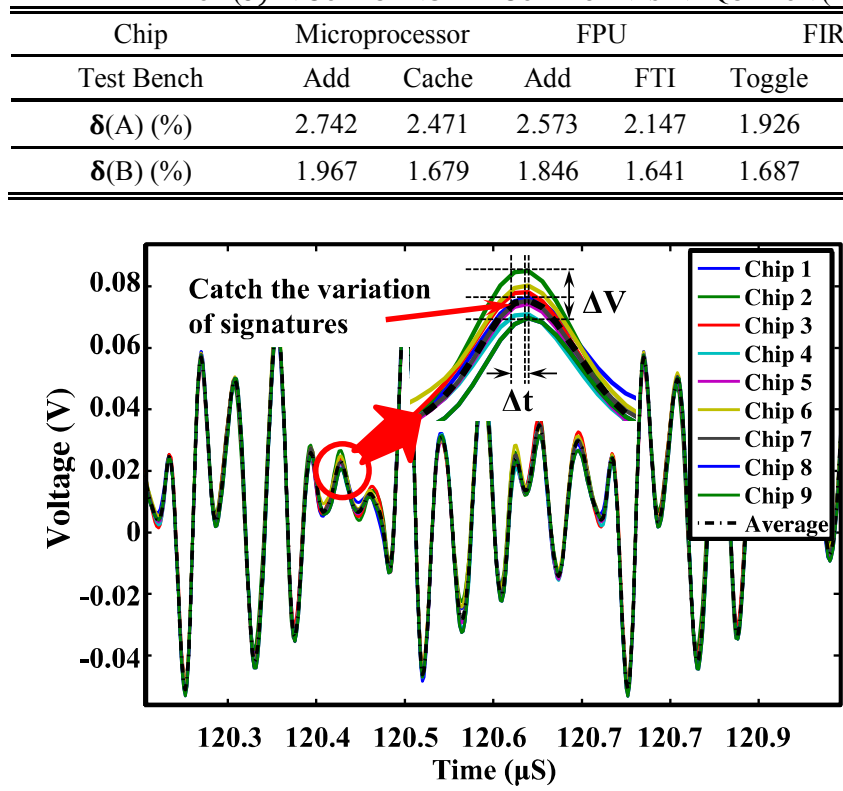

(a)

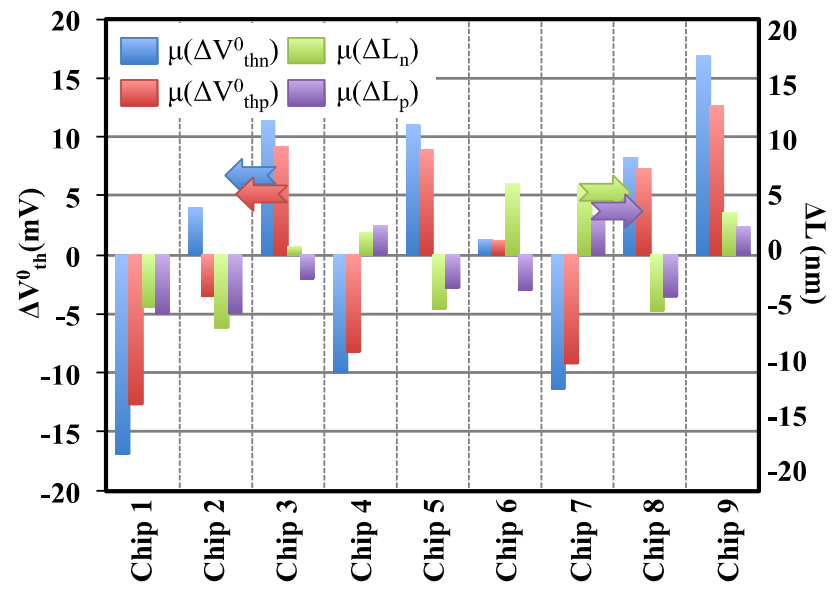

(b)

Fig.7. Measurement and extraction results from the initial signature signal variation. (a) Signature signal at time zero for several microprocessors and (b) extracted process parameters.

\section{EXPERIMENTAL RESULTS}

Our PV and $V_{t h}$ degradation models have been applied to extract PV parameters and NBTI parameters for several microprocessor test chips, which is the same design as one of the simulated circuits. In this case study, the MOSFET gate lengths ranged from $90 \mathrm{~nm}$ to $500 \mathrm{~nm}$ and a wide gate width of $5 \mu \mathrm{m}$ was used. The nominal threshold voltage was $0.4 \mathrm{~V}$ at $25 \mathrm{C}^{\circ}$. We considered supply voltages of $1.4 \mathrm{~V}, 1.6 \mathrm{~V}$, and $1.8 \mathrm{~V}$, and temperatures of $40^{\circ} \mathrm{C}, 60^{\circ} \mathrm{C}, 80^{\circ} \mathrm{C}, 100^{\circ} \mathrm{C}$, and $120^{\circ} \mathrm{C}$. Our purpose is to show that shifts in amplitude and delay are measureable, and hence can be used to extract the shift in threshold voltage. Also, we applied a DC input signal to stress the chips in order to avoid the HCI degradation effect. The dynamic test bench is applied during testing so that ground bounce can be observed.
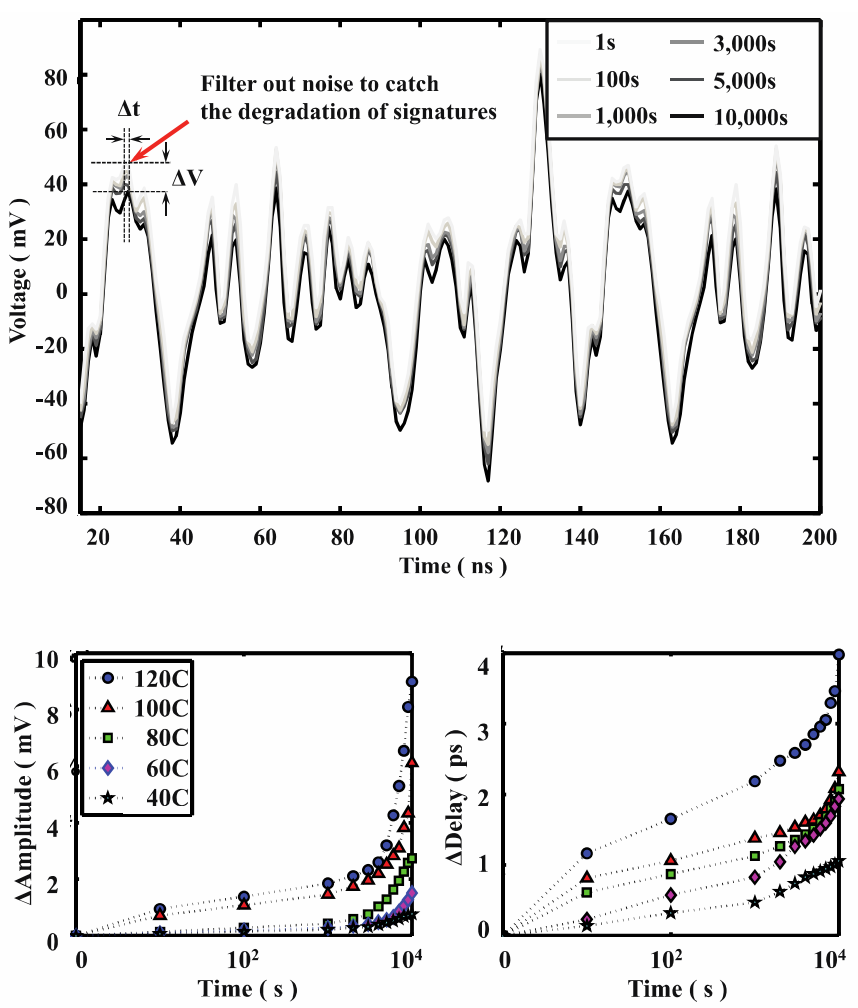

(b)

Fig. 8. Measurement result of signature signal degradation. (a) Degradation of the ground signature signal when tested with a supply voltage of $1.6 \mathrm{~V}$ and a temperature of $100^{\circ} \mathrm{C}$. (b) Degradation of amplitude and delay extracted from the ground signature signal for the circuit tested with a supply voltage of $1.6 \mathrm{~V}$.

\section{A. Data Collection and Computation of PV Parameters and Threshold Voltage Degradation}

As noted in the previous section, the time zero ground signature signal was recorded with an oscilloscope for extraction of process parameters. Several initial ground signals are shown in Fig. 7(a). We measured the ground voltage signature from nine chips. The average of the nine signatures is assumed to be the nominal case. Using equation (5), the calculated process parameter shifts from nominal are shown in Fig. 7(b).

Next, after intervals of voltage and temperature stress, we monitored the ground signal. The NBTI-induced ground voltage was recorded by an oscilloscope, and $\Delta$ Amplitude and $\Delta$ Delay were computed at peaks in the ground signal. In Fig. $8(\mathrm{a})$, we capture the voltage signature degradation as a function of time for different input voltages and temperatures. The extracted degradation data is shown in Fig. 8(b). Using equation (6), the extracted $V_{\text {thp }}$ drift data are shown in Fig. 9. These degradation results are similar to data in the literature [28],[32],[35].

We have used a sampling oscilloscope and have used the "average" function and "bandpass filter" function to filter out the noise and jitter. These functions use 512 samples of the same time point to generate a stable ground signal. The sampling is every 0.2 picosecond, which is sufficient to see the delay degradation. The resulting signal is then processed for amplitude and peak detection. 


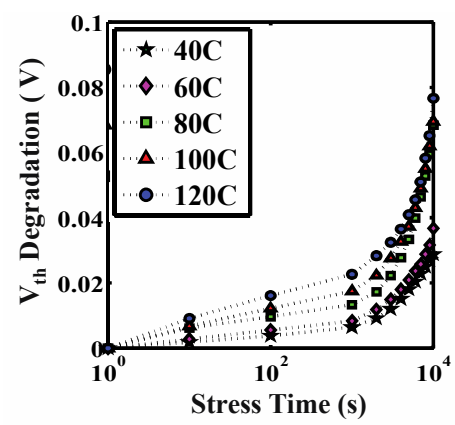

(a)

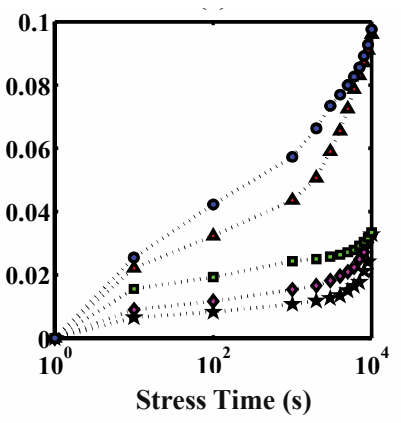

(b)
Fig. 9. The threshold voltage degradation for the microprocessor derived by Equation (6). (a) Input voltage 1.4V. (b) Input voltage $1.8 \mathrm{~V}$.

\section{B. Performance Degradation Analysis}

In order to further study the joint impact of NBTI and PV on the microprocessor, we have extracted the critical paths through static timing analysis (STA). A detailed explanation of the methodology is given in [36],[37].

The models we have developed are for test conditions. They are scaled to use conditions by adjusting $\varphi\left(T, E_{F}\right)$. The lifetime is the time until the system suffers from timing violations. NBTI and PV impact these timing margins. Lifetime is a function of frequency because higher operating frequencies have smaller timing margins.

Fig. 10 shows the estimated lifetime of each chip as a function of operating frequency. It can be seen that each chip has unique values for PV and NBTI parameters. PV affects the lifetime intercept (A in Equation (1)). As a result, some chips start out more vulnerable to NBTI because of PV. The extracted NBTI parameters primarily affect the slope. This causes the lifetime of some chips to be more sensitive to frequency.

\section{CONClusion}

This paper presents two models, one is for extraction of initial process parameters, and the other is for extraction of system-level NBTI parameters. We used simulation to develop an equation to extract the difference in the MOS length and threshold voltage from nominal using the initial ground signature. Also, the ground signature is used for extraction of an average threshold voltage shift, from which NBTI failure rate parameters are computed. The method involves measuring shifts in amplitude and delay. The ability to measure shifts in the ground signal has been demonstrated experimentally.

This approach enables the estimation of process variation aware system reliability under BTI degradation on a chip-bychip basis. It determines an average threshold voltage shift, from which wearout distribution parameters are estimated. This shift depends on actual usage. Hence, if the threshold voltage is monitored periodically over time, lifetime can be estimated while taking into account actual usage. The model can then be used to forecast lifetime based on similar usage. Alternatively, if chips are stressed periodically, the proposed methodology can be used to estimate the remaining lifetime.

Future work will explore extensions that involve estimation of PBTI in nMOS devices in technologies beyond $90 \mathrm{~nm}$.

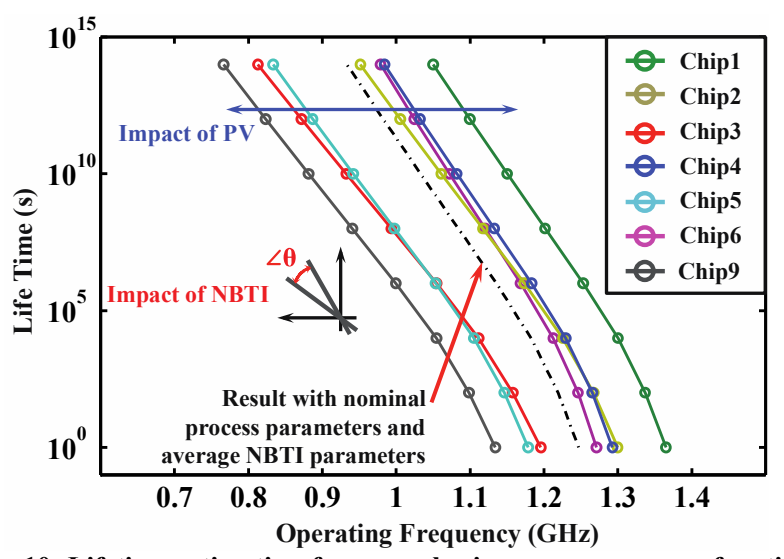

Fig. 10. Lifetime estimation for several microprocessors as a function of operating frequency.

\section{ACKNOWLEDGMENT}

The authors thank the Defense Advanced Research Projects Agency (DARPA) under grant HR0011-11-1-0011 for support.

\section{REFERENCES}

[1] D. K. Schroder and J.A. Babcokc, "Negative bias temperature instability: Road to cross in deep submicron silicon semiconductor manufacturing," $J$. Appl. Phys., vol. 94, no. 1, pp. 1-18, July 1, 2003.

[2] S. Bhardwaj, W. Wang, R. Vattikonda, Y, Cao, and S. Vrudhula, "Predictive modeling of the NBTI effect for reliable design," in Proc. Custom Integrated Circuits Conf., 2006, pp. 189-192.

[3] D. K. Schroder, "Negative bias temperature instability: What do we understand?" Microelectron. Reliab., vol. 47 no. 6, pp. 841-852, Jun. 2007.

[4] H. Reisinger, T. Grasser, W. Gustin, and C. Schlnder, "The statistical analysis of individual defects constituting NBTI and Its implications for modeling DC- and AC-stress," in Proc. IRPS, May 26, 2010, pp. 7-15.

[5] P. Lenahan, P. Campbell, T. Krishnan, and S. Krishnan, "A model for NBTI in nitrided oxide MOSFETs which does not involve hydrogen or diffusion," IEEE Trans. Device Mater. Rel., vol. 11, no. 2, pp. 219-226, 2011.

[6] D. Ang, Z. Teo, T. Ho, and C. Ng, "Reassessing the mechanisms of negativebias temperature instability by repetitive stress/relaxation experiments," IEEE Trans. Device Mater. Rel., vol. 11, no. 1. pp. 19-34, 2011.

[7] H. Reisinger, O. Blank, W. Heinrigs, A. Mhlhoff, W. Gustin, and C. Schlnder, "Analysis of NBTI degradation and recovery-behavior based on ultra fast Vth-measurements," in Proc.IRPS., 2006, pp. 448-453.

[8] V. Huard, M. Denais, and C. Parthasarathy, "NBTI degradation: From physical mechanisms to modeling," Microelectron. Reliab., 2006, vol. 46, no. 1, pp. 1-23.

[9] J. Fang and S. Sapatnekar. "The Impact of BTI Variations on Timing in Digital Logic Circuits." IEEE Trans. Device Mater. Rel., 2013, vol. 13, no. 1, pp. 277-286.

[10] H. Luo, Y. Wang, K. He, R. Luo, H. Yang, and Y. Xie, "A novel gate-level NBTI delay degradation model with stacking effect." in Integrated Circuit and System Design. Power and Timing Modeling, Optimization and Simulation, Springer, 2007, pp. 160-170.

[11] W. Wang, S. Yang, S. Bhardwaj, S. Vrudhula, F. Liu, and Y. Cao, "The impact of NBTI effect on combinational circuit: Modeling simulation, and analysis," IEEE Trans. VLSI, vol. 18, no. 2, pp. 173-183, Feb. 2010.

[12] S. Kiamehr, F. Firouzi, and M.B. Tahoori, "Aging-aware timing analysis considering combined effects of NBTI and PBTI," in Proc. Int'l Symp. on Quality Electronics Design, 2013, pp. 53-59.

[13] S.V. Kumar, C.H. Kim, and S.S. Sapatnekar, "Adaptive techniques for overcoming performance degradaiton due to aging in CMOS circuits," IEEE Trans. VLSI, vol. 19, no. 4, pp. 603-614, April 2011.

[14] J. Keane, X. Wang, D. Persaud, and C.H. Kim, "An all-in-one silicon odometer for separately monitoring HCI, BTI, and TDDB," IEEE J. SolidState Circuits, vol. 45, no. 4, pp. 817-829, April 2010.

[15] J.W. Tschanz, J.T. Kao, S.G. Narendra, R. Nair, D.A. Antoniadis, A.P. Chandrakasan, and V. De, "Adaptive body bias for reducing impacts of dieto-die and within-die parameter variations on microprocessor frequency and leakage," IEEE J. Solid-State Circuits, vol. 37, no. 11, pp. 1396-1402, Nov. 2002 . 
[16] S. Narendra, A. Keshavarzi, B.A. Bloechel, S. Borkar, and V. De, "Forward body bias for microprocessors in 130-nm technology generation and beyond," IEEE J. Solid-State Circuits, vol. 38, no. 5, pp. 696-701, May 2003.

[17] S. Arasu, M. Nourani, J.M. Carulli, K.M. Butler, and V. Reddy, "A designfor-reliability approach based on grading library cells for aging effects," in Proc. Int. Test Conf., 2013.

[18] F. Ahmed and L. Milor, "Via weraout detection with on chip monitors," in Proc. Int. Workshop on Advances in Sensors \& Interfaces, 2009, pp. 156-161.

[19] X. Wang, M. Tehranipoor, S. George, D. Tran, and L. Winemberg, "Design and analysis of a delay sensor applicable to process/environmental variations and aging measurements," IEEE Trans. VLSI, vol. 20, no. 8, pp. 1405-1418, Aug. 2012.

[20] S. Das, D. Roberts, S. Lee, S. Pant, D. Blaauw, T. Austin, K. Flautner, and T. Mudge, "A self-tuning DVS processor using delay-error detection and correction," IEEE J. Solid-State Circuits, vol. 41, no. 4, April 2006, pp. 792804.

[21] J.C. Vazquex, V. Champac, I.C. Teixeira, M.B. Santos, and J.P. Teixeira, "Programmable aging sensor for automotive safety-critical applicaitns," in Proc. Design Automation and Test in Europe, 2010, pp. 618-621.

[22] A. T. Krishnan, V. Reddy, S. Chakravarthi, J. Ropdriguez, S. John, and S. Krishnan, "NBTI impact on transistor and circuit: Models, mechanisms, and scaling effect," in IEDM Tech. Dig., 2003, pp. 1-4.

[23] A. van der Wel, E. A. M. Klumperink, J. S. Kolhatkar, E. Hoekstra, M. S. Snoeij, C. Salm, H. Wallinga, and B. Nauta, "Low-frequency noise phenomena in switched MOSFETs," IEEE J. Solid-State Circuits., 2007, vol. 42 , no. 3, pp. 540-550.

[24] M. J. Kirton and M. J. Uren, "Noise in solid-state microstructures: A new perspective on individual defects, interface states and low- frequency (1/f) noise," J. Advances in Physics., 1989, vol. 38, no. 4, pp. 367-468.

[25] S. Machlup, "Noise in semiconductors: Spectrum of a two-parameter random signal," J. Appl. Phys., vol. 25, no. 3, pp. 341-343, Mar. 1954.

[26] J. Fang and S. Sapatnekar. "The Impact of BTI Variations on Timing in Digital Logic Circuits.” IEEE Trans. Device Mater. Rel., 2013, vol. 13, no. 1, pp. 277-286.
[27] R. da Silva and G. Wirth, "Logarithmic behavior of the degradation dynamics of metal oxide semiconductor devices," J. Stat. Mech., Theory Exp., no. 4, pp. 04-025, Apr. 2010.

[28] G.I. Wirth, R. da Silva, and B. Kaczer, "Statistical Model for MOSFET Biast Temperature Instability Component Due to Charge Trapping," IEEE Trans. Electron Devices Aug. 2011, vol. 58, no. 8, pp. 2743-2751

[29] G. Wirth, R. da Silva, P. Srinivasan, J. Krick, and R. Brederlow, "Statistical model for MOSFET low-frequency noise under cyclo- stationary conditions," in IEDM Tech. Dig., 2009, pp. 1-4.

[30] B. Kaczer, T. Grasser, P. J. Roussel, J. Franco, R. Degraeve, L. Ragnarsson, E. Simoen, G. Groeseneken, and H. Reisinger, "Origin of NBTI variability in deeply scaled pFETs," in Proc. Int. Reliability Physics Symp., 2010, pp. 2632.

[31] A.T. Krishnan, F. Cano, C. Chancellor, V. Reddy, Z. Qi, P. Jain, J. Carulli, J. Masin, S. Zuhoski, S. Krishnan, and J. Ondrusek, "Product drift from NBTI: Guardbanding, circuit and statistical effects," in IEDM Tech. Dig., 2010, pp. 4.3.1.-4.3.4.

[32] T. Grasser, and B. Kaczer, "Evidence that two tightly coupled mechanisms are responsible for negative bias temperature instability in oxynitride MOSFETs," IEEE Trans. Electron Devices, vol. 56, no. 5, May 2009.

[33] G.E.P. Box, W.G. Hunter, and J.S. Hunger, Statistics for Experimenters, John Wiley \& Sons Inc., 1978.

[34] G.S. May and C.J. Spanos, Fundamentals of Semiconductor Manufacturing and Process Control, John Wiley \& Sons Inc., 2006.

[35] J.B. Velamala, K.B. Sutaria, T. Sato, and Y. Cao, "Aging Statistics based on Trapping/Detrapping: silicon evidence, modeling and long-term prediction,' in Proc. Int. Reliability Physics Symp., 2012, pp. 2F.2.1- 2F.2.5.

[36] C.-C. Chen, and L. Milor. "System-level modeling and reliability analysis of microprocessor systems." In IEEE Int. Workshop Advances in Sensors \& Interfaces, 2013, pp. 178-183.

[37] C.-C. Chen, T. Liu, S. Cha, and L. Milor, "System-level modeling of microprocessor reliability degradation due to BTI and HCI," in Proc. Int. Reliability Physics Symp. , 2014. 